Eduardo Moreira Novaes Neto ${ }^{\mathrm{a}}$ (iD) https://orcid.org/0000-0002-4619-8951

Tania Maria de Araújo

iD https://orcid.org/0000-0003-2766-7799

Camila Carvalho Sousa ${ }^{\mathrm{a}}$

(D) https://orcid.org/0000-0002-6139-0984

a Universidade Estadual de Feira de Santana, Departamento de Saúde.

Feira de Santana, BA, Brasil.

Contato:

Tânia Maria de Araúijo

E-mail:

araujo.tania@uefs.br

Este estudo recebeu apoio financeiro da Fundação de Amparo à Pesquisa do Estado da Bahia - FAPESB (Processos no SUS0024/2009 eSUS0022/2014) e do Conselho Nacional de Desenvolvimento Cientifico e Tecnológico - CNPq (Processo 480611/2010-6).

Os autores declaram que não há conflitos de interesses.

Os autores informam que o trabalho não foi apresentado em eventos científicos e que não é baseado em dissertação ou tese.
Recebido: 10/08/2018

Revisado: 18/06/2019

Aprovado: 26/06/2019

\section{Hipertensão Arterial e Diabetes Mellitus entre trabalhadores da saúde: associação com hábitos de vida e estressores ocupacionais}

\author{
Hypertension and diabetes in healthcare workers and their \\ association to life habits and occupational stressors
}

\section{Resumo}

Objetivo: avaliar a associação entre hábitos de vida e aspectos psicossociais do trabalho com a ocorrência de Hipertensão Arterial Sistêmica (HAS) e Diabetes Mellitus (DM), entre os trabalhadores da saúde. Métodos: estudo epidemiológico de corte transversal. Selecionamos os participantes por amostragem aleatória e estratificada. Foram estudados 3.084 trabalhadores da Atenção Básica e dos serviços da Média Complexidade atuantes em cinco municípios da Bahia. As variáveis de desfecho foram definidas segundo a morbidade autorreferida. Os hábitos de vida estudados foram: prática de atividade física, ingestão de bebida alcoólica, tabagismo e prática de atividades de lazer. Para a avaliação dos aspectos psicossociais do trabalho, utilizou-se o Job Content Questionnaire. Resultados: a prevalência de HAS e DM entre os trabalhadores foi de 18,0\% e 4,0\%, respectivamente. Após o ajuste por sexo e idade, o trabalho passivo associou-se à HAS e ao DM, e o tabagismo associou-se apenas ao DM. Conclusão: o hábito de fumar e o trabalho realizado sob condições psicossociais desfavoráveis (trabalho passivo) devem receber atenção especial na prevenção de HAS e DM. É necessário estimular a adoção de hábitos de vida saudáveis e de condições de trabalho adequadas para prevenir o adoecimento crônico e proteger a saúde dos trabalhadores.

Palavras-chave: diabetes mellitus; hipertensão arterial; fatores psicossociais do trabalho; doenças crônicas; saúde do trabalhador.

\begin{abstract}
Objective: to evaluate the association between life habits and psychosocial factors at work with the presence of hypertension and diabetes mellitus in healthcare workers. Methods: in this cross-sectional study, we used randomized and stratified sampling to select the participants. A total of 3,804 individuals working in Primary Healthcare and medium-complexity services of five municipalities in the State of Bahia, Brazil, participated. The outcome variables were defined according to self-reported morbidity. We analyzed the following life habits: physical activities practice, alcoholic drinks ingestion, smoking and engagement in leisure activities. We used the Job Content Questionnaire to evaluate psychosocial factors at work. Results: the prevalence of hypertension and diabetes was $18 \%$ and $4 \%$, respectively. After age and sex adjustments, passive labor was associated to both illnesses, and smoking only with diabetes. Conclusion: smoking and working under unfavorable psychosocial conditions (passive labor) must be the target of hypertension and diabetes prevention. Healthy life habits and proper working conditions must be stimulated to prevent chronic diseases and to protect the workers' health.
\end{abstract}

Keywords: diabetes mellitus; hypertension; psychosocial factors; chronic diseases; occupational health. 


\section{Introdução}

Doenças crônicas são multifatoriais; diversos fatores de risco podem contribuir para ocorrência desses agravos, incluindo o estilo de vida e exposições ocupacionais ${ }^{1}$. Dentre esses fatores, destacam-se os estressores ocupacionais e hábitos de vida negativamente associados à preservação e promoção da saúde, como a ausência de atividade física e de lazer e o consumo de álcool e de tabaco. Assim, esses fatores podem comprometer a saúde das pessoas, produzindo situações de maior exposição aos agravos crônicos ${ }^{2}$.

Evidências consistentes têm fortalecido a hipótese de que os mecanismos relacionados ao adoecimento crônico, como, por exemplo, da hipertensão arterial sistêmica (HAS) e da diabetes mellitus (DM), podem estar relacionados à vivência de situações estressoras no trabalho e às alterações no estilo de vida decorrentes da organização, divisão e condições de trabalho ${ }^{3,4}$.

Doenças cardiovasculares destacam-se no perfil de morbimortalidade da população brasileira. A HAS e o DM, por sua vez, constituem destacados fatores de risco para as doenças cardiovasculares. Adicionalmente, esses dois agravos respondem como causas importantes de hospitalizações no Brasil e no mundo, representando problema de saúde pública de significativa relevância. Assim, medidas voltadas à redução de HAS e DM, com a prevenção, manejo e cuidado adequado das pessoas com esses agravos podem gerar impactos significativos no sistema público de saúde ${ }^{5}$. Identificar fatores associados à ocorrência ou ao agravamento de HAS e DM são centrais na promoção e manutenção de boas condições de saúde das populações. Neste estudo analisamos fatores potencialmente associados à HAS e DM.

Características do trabalho podem influenciar comportamentos e estilo de vida ${ }^{6,7}$. Desse modo, fatores como longas jornadas, múltiplos empregos e trabalho em turnos podem dificultar o estabelecimento de hábitos de vida saudáveis, especialmente com relação à regularidade e qualidade da alimentação consumida e à prática de exercícios físicos. No caso dos trabalhadores da saúde, soma-se a esses fatores, a própria natureza da atividade no setor que os confronta, diariamente, com o estresse e a ansiedade ${ }^{2}$.

Como o local de trabalho é muitas vezes o fator gerador do estresse, as chances de desenvolvimento de doenças aumentam em função do tipo de atividade que o indivíduo executa, bem como os diversos fatores que tornam o ambiente de trabalho insalubre ${ }^{8}$. Dentre os fatores estressores no ambiente ocupacional, destacam-se os relacionados à organização do trabalho, como os aspectos psicossociais ${ }^{9}$.
Neste estudo são focalizados os hábitos de vida e os aspectos psicossociais do trabalho e a relação destes com HAS e DM. Assim, pergunta-se: os hábitos de vida e os aspectos psicossociais do trabalho estão associados a HAS e DM entre trabalhadores da saúde?

Os objetivos deste estudo são estimar a prevalência de hipertensão arterial sistêmica e diabetes mellitus e avaliar a associação entre hábitos de vida e aspectos psicossociais do trabalho com a ocorrência de hipertensão arterial sistêmica e diabetes mellitus entre os trabalhadores de saúde da atenção básica e de média complexidade em cinco municípios da Bahia.

Considerando o impacto do trabalho sobre o adoecimento crônico, e que este pode estar associado à ocorrência de HAS e de DM - morbidades com frequências de grande magnitude nas populações -, os resultados produzidos poderão contribuir para a elaboração de medidas de intervenção com potencial de reduzir a frequência desses agravos.

\section{Métodos}

O presente trabalho é parte de um estudo epidemiológico de corte transversal conduzido em cinco municípios baianos (Feira de Santana, Itabuna, Jequié, Salvador e Santo Antônio de Jesus), com trabalhadores de saúde dos serviços de média complexidade e da atenção básica nos anos de 2011 e 2012.

No estudo multicêntrico, a amostra foi definida em etapas sucessivas: a) obteve-se as listas nominais de todos os trabalhadores em atividade nos serviços de saúde da atenção básica e da média complexidade, fornecidas pelas Secretarias de Saúde dos municípios estudados. As listas continham dados de sexo, idade, ocupação e local de trabalho e foram comparadas com dados disponíveis no Cadastro Nacional de Estabelecimentos de Saúde (CNES); b) estimou-se o tamanho amostral com base no total da população e nos diferentes desfechos de saúde; c) definido o tamanho amostral, estratificou-se a amostra em três níveis: área geográfica, nível de assistência (atenção básica e média complexidade) e grupo ocupacional. A composição da amostra foi definida com base na participação percentual de cada grupo por nível de estratificação estabelecido; d) estimou-se o tamanho amostral em cada estrato; seguindo-se o sorteio dos trabalhadores para comporem a amostra (o sorteio foi feito com base em listagem de números aleatórios do EpiInfo 6.04d). Após o sorteio, os trabalhadores foram contatados nos seus locais de trabalho, sendo feitas até três visitas para a realização da entrevista.

Em cada município foi formada uma equipe de pesquisa local, ficando como responsáveis pela 
condução do estudo em cada local as pesquisadoras das universidades e dos serviços de saúde envolvidos no projeto A gestão do projeto incluiu a coordenação geral e as coordenações de cada município integrante do projeto.

Para padronizar os procedimentos metodológicos adotados em cada local, foi elaborado um Manual de Procedimentos e Condutas e foram realizadas oficinas para o treinamento e preparo dos entrevistadores para a coleta dos dados. Nesta etapa foram abordados múltiplos aspectos, entre os quais se destaca a construção de estratégias para: a) a entrada nos locais de trabalho; b) a sensibilização para a participação no estudo (campanha informativa e de divulgação do estudo) e c) a abordagem do profissional (contato inicial e entrevista).

O questionário foi construído com base na revisão de literatura e estruturado em blocos abordando os seguintes aspectos: I. Dados sociodemográficos; II. Informações gerais sobre o trabalho; III. Informações sobre o ambiente de trabalho e situação vacinal dos trabalhadores; IV. Características psicossociais do trabalho; V. Atividades domésticas e hábitos de vida; VI. Capacidade para o trabalho; VII. Aspectos relacionados à saúde; e VIII. Atos de violência dentro e/ou fora do trabalho. O questionário foi previamente testado em estudo piloto realizado no município de Santo Antônio de Jesus; outros estudos feitos em outros contextos utilizaram esse mesmo questionário ${ }^{10-12}$.

Os critérios de inclusão do estudo multicêntrico foram: estar em efetivo exercício profissional, aceitar participar da pesquisa e ter tempo mínimo de seis meses de trabalho na unidade de saúde. Os trabalhadores não encontrados, após três tentativas ou os que se recusaram a participar do estudo ou aqueles que estavam de licença durante o período de coleta de dados foram substituídos, respeitando-se a área geográfica, o nível de complexidade do serviço, o grupo ocupacional e o sexo. Neste estudo, também se adotou esses mesmos critérios de inclusão e exclusão.

Para verificar se o estudo multicêntrico tinha poder para avaliar a associação entre hábitos de vida, aspectos psicossociais e HAS e DM, uma vez que a amostra não havia sido calculada com essa finalidade, estimou-se novamente o tamanho amostral necessário, utilizando-se como desfecho de interesse a HAS, por apresentar, com base na literatura revisada $^{13}$, maior prevalência (24\%) do que a DM.

O cálculo foi realizado utilizando o software OpenEpi, versão $3.03^{\mathrm{a}}$, com base nos seguintes parâmetros: população total de trabalhadores de saúde dos cinco municípios ( $\mathrm{N}=4.278)$, prevalência do evento de interesse de $24,0 \%^{13}$, com intervalo de confiança de $95,0 \%$ e erro absoluto de $2 \%$. Acrescentando-se 20\% devido a possíveis perdas/ recusas, obteve-se o número mínimo estimado, para compor a amostra, de 1.492 trabalhadores(as). Neste estudo, foram investigados todos os 3.084 trabalhadores alcançados pelo estudo multicêntrico, atribuindo ao presente trabalho poder suficiente para a análise pretendida.

As variáveis de desfecho foram definidas segundo morbidade autorreferida, obtidas por meio das questões a) possui diagnóstico médico de hipertensão arterial? A resposta afirmativa foi considerada como caso de HAS; b) possui diagnóstico médico de diabetes mellitus? A resposta afirmativa foi considerada como caso de DM.

Como variáveis de exposição foram consideradas: a) hábitos de vida: prática de atividade física, consumo de bebidas alcoólicas, tabagismo e realização de atividades de lazer; b) aspectos psicossociais do trabalho: estressores ocupacionais avaliados por meio do Job Content Questionnaire (JCQ) proposto por Karasek ${ }^{14}$.

O JCQ é um instrumento que avalia a experiência psicossocial no trabalho. Destacam-se duas dimensões centrais para investigação do estresse laboral: a) a demanda psicológica relacionada às exigências mentais enfrentadas pelo trabalhador durante a execução de suas atividades laborais (ex.: ritmo de trabalho, facilidade ou dificuldade em realizá-lo) e b) o controle do trabalhador sobre as atividades laborais que exerce, incluindo sua autonomia no processo de decisão. A partir da combinação da demanda psicológica e do controle sobre o trabalho, é obtido o modelo demanda-controle (MDC), composto por quatro situações específicas de trabalho que configuram riscos à saúde do trabalhador ${ }^{15}$.

Considerando-se a mediana como ponto de corte para a definição das categorias alto(a) e baixo(a), as experiências de trabalho foram classificadas, segundo o MDC, em: baixa exigência (alto controle/baixa demanda); trabalho ativo (alto controle/alta demanda); trabalho passivo (baixo controle/baixa demanda) e alta exigência (baixo controle/alta demanda) ${ }^{15}$.

Como covariáveis, foram analisadas as características sociodemográficas (sexo, idade, raça/cor da pele, renda, número de filhos e situação conjugal) e as características gerais do trabalho (tempo de trabalho no emprego atual, tipo de vínculo empregatício, jornada de trabalho e turno de trabalho).

Foram realizadas análises univariada, bivariada e multivariada. Na análise univariada foram estimadas as frequências brutas e relativas para cada uma das variáveis selecionadas.

$\mathrm{Na}$ análise bivariada foram estimadas as razões de prevalência, intervalos de confiança $(\mathrm{IC}=95 \%)$ e 
valores de $p$ pelo teste qui-quadrado de Pearson para verificar a associação entre as variáveis de exposição (hábitos de vida e aspectos psicossociais do trabalho) e a prevalência de HAS e DM. O nível de significância estatística adotado foi de 5\%. Nesta etapa foram utilizados os programas Statistical Program for the Social Sciences 24.0 (SPSS 24.0) ${ }^{17}$ e OpenEpi 3.03 ${ }^{\mathrm{a} 14}$.

Para investigar confundimento, foram consideradas variações entre a medida de associação bruta e a ajustada por cada covariável de interesse ${ }^{16}$ iguais ou maiores que $20 \%$, bem como evidências teóricas de relevância de fatores para os desfechos considerados (HAS e DM) ${ }^{3,4}$. Como nenhuma das covariáveis investigadas (sexo, idade, raça/cor, jornada de trabalho e turno de trabalho) apresentou variações superiores a $20 \%$ na análise de confundimento, utilizou-se o conhecimento teórico para seleção das variáveis de confusão. Assim, as variáveis sexo e idade foram acrescidas ao modelo final para ajuste.

Para a análise multivariada, realizou-se regressão logística não condicional. O nível de significância para entrada no modelo multivariado foi $25 \%$. As associações foram consideradas estatisticamente significantes ao nível de 5\%. Com base no modelo final obtido, as razões de prevalência foram estimadas utilizando Regressão de Possion. Os dados foram analisados no Data Analysis and Statistical Software (STATA), versão 10.0.

O diagnóstico de qualidade do modelo final foi avaliado pelo teste de bondade de ajuste do modelo (Hosmer e Lemeshow), Curva ROC e padrão de observações influentes.

Os protocolos de pesquisa foram avaliados e aprovados pelo Comitê de Ética em Pesquisa com Seres Humanos da Universidade Estadual de Feira de Santana, Bahia, sob parecer $n^{\circ} 081 / 2009$. Os profissionais selecionados para o estudo foram informados dos objetivos da pesquisa e após concordarem em participar, assinaram o Termo de Consentimento Livre e Esclarecido (TCLE).

\section{Resultados}

Dentre os 3.084 entrevistados, a maioria era de mulheres $(78,0 \%)$, se autodeclarou pretos(as) ou pardos(as) $(70,7 \%)$, com idade superior a 35 anos $(58,1 \%)$, com filhos $(68,6 \%)$; dentre estes, $73,0 \%$ possuíam um ou dois filhos. O nível de escolaridade de maior percentual foi o nível superior $(43,0 \%)$, seguido do nível fundamental e médio (40,2\%). Sobre a situação conjugal, 57,3\% possuíam companheiro(a). Quando indagados sobre sua renda mensal, $73,1 \%$ relataram receber mais que dois salários mínimos.

De acordo com as características ocupacionais, $58,5 \%$ dos trabalhadores possuíam vínculo empregatício do tipo efetivo/concursado. A maioria (65,2\%) tinha até 10 anos de tempo de trabalho. No que diz respeito a jornada de trabalho, percentual elevado $(77,2 \%)$ relatou trabalhar até 40 horas semanais. O turno de trabalho mais frequente foi o diurno $(79,8 \%)$, que correspondia aos trabalhos no turno da manhã e da tarde.

Sobre os hábitos de vida dos trabalhadores, 56,1\% relataram não praticar atividade física. Quando questionados sobre atividades de lazer, 83,6\% relataram usufruir de tais atividades. Dentre as atividades de lazer mais frequentes, encontravam-se: assistir televisão, ouvir rádio e frequentar atividades culturais e sociais. No que diz respeito ao tabagismo, 6,0\% eram fumantes. Sobre o consumo de bebidas alcoólicas, 39,1\% referiram este hábito (Tabela 1).

Em relação aos aspectos psicossociais do trabalho, 21,8\% dos trabalhadores foram classificados no grupo de alta exigência, estrato considerado o de maior exposição às condições de trabalho que geram estresse (baixo controle sobre o trabalho e alta demanda psicológica). No que tange aos desfechos deste estudo, a prevalência autorreferida de hipertensão arterial foi de $18 \%$, enquanto a prevalência do diabetes mellitus foi relativamente menor: 4\% (Tabela 1).

Na análise de associação entre hábitos de vida e estressores ocupacionais com HAS (Tabela 2), observou-se associação positiva, estatisticamente significante, apenas com estressores ocupacionais (trabalho passivo) $(\mathrm{RP}=1,24$; $\mathrm{IC}=1,007-2,534)$.

A Tabela 3 mostra a análise de associação entre hábitos de vida e estressores ocupacionais e DM. Foi observada associação positiva, estatisticamente significante, de acordo com os parâmetros adotados, com tabagismo $(\mathrm{RP}=2,34 ; \mathrm{IC}=1,397-3,944) \mathrm{e}$ trabalho passivo $(\mathrm{RP}=1,70 ; \mathrm{IC}=1,068-2,710)$.

As Tabelas 4 e 5 apresentam os resultados da análise de regressão logística não condicional. Em relação a HAS, permaneceu no modelo final o trabalho passivo (RP: 1,30; IC: 1,002 - 1,677). Dentre o conjunto de variáveis analisadas, permaneceram associadas ao DM: tabagismo (RP: 2,65; IC: $1,498-4,679$ ) e trabalho passivo (RP: 1,72; IC: 1,051-2,802). 
Tabela 1 Prevalência de hipertensão arterial e diabetes mellitus e caracterização do trabalho considerando modelo demanda-controle e hábitos de vida entre trabalhadores da saúde da atenção básica e dos serviços de média complexidade nos municípios de Feira de Santana, Itabuna, Jequié, Salvador e Santo Antônio de Jesus. Bahia, Brasil, 2011-2012

\begin{tabular}{|c|c|c|}
\hline Variáveis & $n$ & $\%$ \\
\hline \multicolumn{3}{|l|}{ Hipertensão Arterial $(n=3.029)$} \\
\hline Sim & 546 & 18,0 \\
\hline Não & 2.483 & 82,0 \\
\hline \multicolumn{3}{|l|}{ Diabetes Mellitus ( $n=3.013)$} \\
\hline Sim & 121 & 4,0 \\
\hline Não & 2.892 & 96,0 \\
\hline \multicolumn{3}{|c|}{ Modelo Demanda-Controle $(\mathrm{n}=2.902)^{*}$} \\
\hline Baixa exigência & 850 & 29,3 \\
\hline Trabalho ativo & 633 & 21,8 \\
\hline Trabalho passivo & 787 & 27,1 \\
\hline Alta exigência & 632 & 21,8 \\
\hline \multicolumn{3}{|l|}{ Atividade Física $(\mathrm{n}=2.652)$} \\
\hline Sim & 1.164 & 43,9 \\
\hline Não & 1.488 & 56,1 \\
\hline \multicolumn{3}{|l|}{ Atividade de Lazer $(\mathrm{n}=3.058)$} \\
\hline Sim & 2.555 & 83,6 \\
\hline Não & 503 & 16,4 \\
\hline \multicolumn{3}{|l|}{ Tabagismo $(n=3.020)$} \\
\hline Sim & 182 & 6,0 \\
\hline Não & 2.838 & 94,0 \\
\hline \multicolumn{3}{|l|}{ Alcoolismo $(n=2.783)$} \\
\hline Sim & 1.089 & 39,1 \\
\hline Não & 1.694 & 60,9 \\
\hline
\end{tabular}

"Houve perda de informação para 182 (5,8\%) trabalhadores. Na variável “modelo demanda-controle”, a demanda é medida por 5 itens e controle por 9 itens. A ausência de resposta a qualquer um desses 14 itens resulta em perda de informação para essa variável. Para diabete mellitus, a perda por não resposta foi de 71 (2,3\%); para hipertensão arterial, 55 (1,7\%); para atividade física, 432 (14,0\%); para lazer, 26 (0,8\%); para tabagismo, 64 (2,1\%); e para alcoolismo, 31 (9,8\%).

Tabela 2 Hipertensão arterial sistêmica segundo os hábitos de vida e os estressores ocupacionais entre os trabalhadores da saúde da atenção básica e dos serviços de média complexidade dos municípios de Feira de Santana, Itabuna, Jequié, Salvador e Santo Antônio de Jesus. Bahia, Brasil, 2011-2012

\begin{tabular}{|c|c|c|c|c|c|}
\hline \multirow{2}{*}{ Hábitos de Vida e Estresse Ocupacional } & \multicolumn{5}{|c|}{ Hipertensão Arterial Sistêmica } \\
\hline & Sim & $\%$ & $R P$ & IC (95\%) & p-valor \\
\hline \multicolumn{6}{|l|}{ Atividade Física $(\mathrm{n}=2.616)$} \\
\hline Sim & 205 & 17,7 & $*$ & & \\
\hline Não & 270 & 18,5 & 1,04 & $0,885-1,229$ & 0,616 \\
\hline \multicolumn{6}{|l|}{ Atividade de Lazer $(n=3.007)$} \\
\hline Sim & 448 & 17,7 & $*$ & & \\
\hline Não & 91 & 19,0 & 1,07 & $0,872-1,310$ & 0,519 \\
\hline \multicolumn{6}{|l|}{ Tabagismo $(n=2.976)$} \\
\hline Sim & 39 & 22,3 & 1,25 & $0,936-1,665$ & 0,139 \\
\hline Não & 500 & 17,9 & * & & \\
\hline \multicolumn{6}{|l|}{ Alcoolismo $(n=2.761)$} \\
\hline Sim & 188 & 17,4 & 0,98 & $0,834-1,1620$ & 0,849 \\
\hline Não & 296 & 17,6 & $*$ & & \\
\hline \multicolumn{6}{|l|}{ Modelo Demanda-Controle $(\mathrm{n}=2.847)$} \\
\hline Baixa exigência & 134 & 16,0 & $*$ & & \\
\hline Trabalho ativo & 113 & 18,1 & 1.13 & $0,901-1,421$ & 0,289 \\
\hline Trabalho passivo & 153 & 19,9 & 1.24 & $1,007-1,534$ & 0,042 \\
\hline Alta exigência & 113 & 18,2 & 1.14 & $0,906-1,428$ & 0,271 \\
\hline
\end{tabular}


Tabela 3 Diabetes mellitus segundo os hábitos de vida e os estressores ocupacioanais entre os trabalhadores da saúde da atenção básica e dos serviços de média complexidade dos municípios de Feira de Santana, Itabuna, Jequié, Salvador e Santo Antônio de Jesus. Bahia, Brasil, 2011-2012

\begin{tabular}{|c|c|c|c|c|c|}
\hline \multirow{2}{*}{ Hábitos de Vida e Estresse Ocupacional } & \multicolumn{5}{|c|}{ Diabetes Mellitus } \\
\hline & $\operatorname{sim}$ & $\%$ & $R P$ & IC (95\%) & p-valor \\
\hline \multicolumn{6}{|l|}{ Atividade Física $(\mathrm{n}=2.602)$} \\
\hline Sim & 43 & 3,7 & * & & \\
\hline Não & 66 & 4,5 & 1,216 & $0,835-1,771$ & 0,309 \\
\hline \multicolumn{6}{|l|}{ Atividade de Lazer $(\mathrm{n}=2.990)$} \\
\hline Sim & 102 & 4,1 & * & & \\
\hline Não & 17 & 3,6 & 0,878 & $0,530-1,453$ & 0,612 \\
\hline \multicolumn{6}{|l|}{ Tabagismo $(n=2.961)$} \\
\hline Sim & 15 & 8,7 & 2,34 & $1,397-3,944$ & 0,001 \\
\hline Não & 104 & 3,7 & * & & \\
\hline \multicolumn{6}{|l|}{ Alcoolismo $(n=2.759)$} \\
\hline Sim & 46 & 4,2 & 1,13 & $0,779-1,640$ & 0,520 \\
\hline Não & 63 & 3,8 & $*$ & & \\
\hline \multicolumn{6}{|l|}{ Modelo Demanda-Controle $(n=2.831)$} \\
\hline Baixa exigência & 28 & 3,4 & * & & \\
\hline Trabalho ativo & 23 & 3,7 & 1,10 & $0,639-1,889$ & 0,733 \\
\hline Trabalho passivo & 43 & 5,7 & 1,70 & $1,068-2,710$ & 0,023 \\
\hline Alta exigência & 24 & 3,9 & 1,16 & $0,678-1,977$ & 0,592 \\
\hline
\end{tabular}

Tabela 4 Análise* de associação entre hipertensão arterial e hábitos de vida e os estressores ocupacioanais entre os trabalhadores da saúde da atenção básica e dos serviços de média complexidade dos municípios de Feira de Santana, Itabuna, Jequié, Salvador e Santo Antônio de Jesus. Bahia, Brasil, 2011-2012

\begin{tabular}{lccc}
\hline \multicolumn{1}{c}{ Variável } & $R P$ & $I C(95 \%)$ & $p$-valor \\
\hline Tabagismo $(\mathrm{n}=2.976)$ & & & 0,305 \\
Sim & 1,23 & $0,830-1,816$ & \\
Não & $*$ & & \\
Modelo Demanda-Controle $(\mathrm{n}=2.847)$ & $*$ & & 0,263 \\
Baixa exigência & 1,17 & $0,888-1,544$ & 0,049 \\
Trabalho ativo & 1,30 & $1,002-1,677$ & 0,301 \\
Trabalho passivo & 1,16 & $0,877-1.527$ & \\
Alta exigência & & & \\
\hline
\end{tabular}

*Modelo ajustado por sexo e idade

Tabela 5 Análise* de associação entre diabetes mellitus e hábitos de vida e os estressores ocupacioanais entre os trabalhadores da saúde da atenção básica e dos serviços de média complexidade dos municípios de Feira de Santana, Itabuna, Jequié, Salvador e Santo Antônio de Jesus. Bahia, Brasil, 2011-2012.

\begin{tabular}{lccc}
\hline \multicolumn{1}{c}{ Variável } & $R P^{*}$ & $I C(95 \%)$ & $p$-valor \\
\hline Tabagismo $(\mathrm{n}=2.976)$ & & & 0,001 \\
Sim & 2,65 & $1,498-4,679$ & \\
Não & $*$ & & \\
Modelo Demanda-Controle $(\mathrm{n}=2.847)$ & $*$ & $0,607-1,897$ & 0,807 \\
Baixa exigência & 1,07 & $1,051-2,802$ & 0,031 \\
Trabalho ativo & 1,72 & $0,666-2,027$ & 0,598 \\
Trabalho passivo & 1,16 & & \\
Alta exigência & & & \\
\hline
\end{tabular}

*Modelo ajustado por sexo e idade 


\section{Discussão}

Os resultados mostraram associação positiva entre os estressores ocupacionais e os hábitos de vida com o adoecimento crônico (HAS e DM) entre os trabalhadores de saúde.

O trabalho passivo (situação combinada de baixo controle e baixas demandas psicológicas) associou-se à HAS, corroborando com dados da literatura que apontam o trabalho nessa situação como desestimulador, capaz de produzir perda de energia vital e de motivação para o trabalho; em estudo realizado no Rio de Janeiro com 1.819 trabalhadoras, que também utilizou o modelo demanda-controle para avaliar os estressores, encontrou-se maior prevalência de HAS no grupo de trabalho passivo ${ }^{17}$.

Exigências excessivas causadas pelas dificuldades cotidianas, incluindo as encontradas no ambiente de trabalho, sobretudo no trabalho em saúde, aumenta o nível de produção e liberação das catecolaminas, como a adrenalina, noradrenalina e cortisol. Esta liberação pode promover o aumento da frequência cardíaca o que, por sua vez, pode contribuir para a elevação da pressão arterial $^{18}$.

$\mathrm{O}$ trabalho passivo associou-se também à DM. O trabalhador sujeito a essa situação trabalha sob condição de baixa demanda psicológica e baixo controle sobre o trabalho. $\mathrm{O}$ trabalho realizado sob essas condições associa-se ao declínio na atividade global e à redução da capacidade de produzir soluções diante das situações e problemas encontrados no ambiente laboral, o que predispõe a vivência de condições nocivas que podem produzir sofrimento e doenças ${ }^{9}$. Ou seja, a exposição cotidiana a essa situação produz quadro de apatia, desmotivação e desinteresse que impactam na saúde das pessoas, em sua qualidade de vida e em suas relações sociais e pessoais. Logo, é uma condição ocupacional desfavorável, que representa risco à saúde física e mental dos trabalhadores.

$\mathrm{O}$ diabetes mellitus relaciona-se com o estresse em função da liberação de níveis de hormônios e neurorreguladores, o que eleva os valores glicêmicos. Sabe-se que o aumento persistente destes níveis glicêmicos pode gerar um estado de hiperglicemia constante que, se for sustentado, pode resultar no diabetes mellitus. Além disso, a ansiedade gerada pelo estresse pode diminuir a tolerância à glicose em pessoas saudáveis, bem como precipitar o diabetes mellitus em pessoas cuja tolerância já está em declínio ${ }^{19}$.

Os trabalhadores da saúde estão frequentemente expostos aos estressores laborais diante da alta demanda dos serviços de saúde, das condições de trabalho e emprego desfavoráveis, das jornadas de trabalho em turnos e dos múltiplos empregos. Todos esses fatores dificultam a prática de hábitos de vida saudáveis, como as atividades físicas, aumentando as chances de desenvolver diabetes mellitus ${ }^{20}$.

Neste estudo, além do trabalho passivo, o tabagismo também permaneceu associado ao DM no modelo final de regressão. A associação entre diabetes mellitus e tabagismo entre trabalhadores da saúde também foi encontrada em outro estudo ${ }^{20}$, da mesma forma que os estressores se destacaram como fator associado à ocorrência de diabetes, como corroborado no estudo de Ortiz e Zanetti ${ }^{19}$.

A hipótese de que a carga de trabalho excessiva, somada ao elevado nível de tensão, torna o trabalhador mais vulnerável à adesão de práticas inadequadas à saúde, como o tabagismo, se confirma ${ }^{13}$. Ou seja, o próprio estresse predispõe o aparecimento de diversos agravos à saúde, além de contribuir para a adesão a hábitos de vida considerados nocivos. Além disto, cabe destacar a relação entre ansiedade e tabagismo descrita na literatura. Segundo resultados obtidos, elevados sintomas de ansiedade social predizem maior uso de tabaco ${ }^{21}$. Assim, sintomas de ansiedade social podem desempenhar importante papel na manutenção do hábito de fumar e na taxa de cessação do tabagismo, aumentando o risco de recaída durante o processo de abandono desse comportamento. Como a hipótese de relação entre aspectos psicossociais do trabalho e ansiedade é fortalecida em vários estudos ${ }^{14,15}$, a potencial interação entre tabagismo e ambiente psicossocial desfavorável no trabalho e sua relação com doenças crônicas, como HAS e DM, constitui tema relevante para exploração em estudos futuros.

Assinala-se também que o trabalhador frequentemente estressado no trabalho, em conjunto com a adoção do tabagismo, apresenta elevado risco de sofrer eventos cardiovasculares ${ }^{3}$, que podem repercutir em diversas consequências para a saúde, além de implicar no risco de morte, elevando-se tanto a morbidade, quanto a mortalidade em decorrência desses eventos.

Diante dos resultados, vale ressaltar que a análise foi realizada com base em desfechos autorreferidos, o que pode ter contribuído para a imprecisão das frequências estimadas de HAS e DM e consequente subestimação ou superestimação das medidas de associação. Apesar de todas as precauções tomadas para evitar vieses, deve-se considerar os vieses inerentes aos estudos transversais (como a causalidade reversa, viés de memória e o viés do trabalhador sadio).

Por fim, apesar das limitações do estudo, os achados obtidos evidenciaram relação entre características psicossociais do trabalho (na situação de trabalho passivo) para DM e HAS e hábitos de vida (tabagismo) para DM. Portanto, os aspectos psicossociais do trabalho 
merecem atenção futura nos programas de prevenção e promoção da saúde entre esses trabalhadores, de modo a reduzir ou eliminar fatores estressores no trabalho. Atenção especial deve ser dada ao grupo exposto a situações de baixa demanda e baixo controle, que foram aqueles com maior frequência de HAS e DM, além da importância de se considerar o hábito de fumar nos programas e ações de intervenção.

\section{Considerações finais}

Este estudo permitiu estimar a prevalência de hipertensão arterial sistêmica e diabetes mellitus entre os trabalhadores da saúde da atenção básica e de média complexidade, bem como suas condições de trabalho e emprego, hábitos de vida e as características psicossociais do trabalho envolvidas.

Verificou-se a associação entre o trabalho passivo (baixo controle sobre o próprio trabalho e baixa demanda) e a ocorrência de hipertensão arterial sistêmica e associação entre trabalho passivo e hábitos de vida (tabagismo) e a ocorrência de diabetes mellitus.

Os resultados obtidos permitiram caracterizar as condições de saúde dos trabalhadores da atenção básica e de média complexidade na Bahia. Isto pode contribuir para a proposição de estratégias preventivas para a saúde do trabalhador da saúde, sobretudo para subsidiar as ações de redução dos estressores ocupacionais relacionados à demanda psicológica e ao nível de controle do trabalho pelo próprio trabalhador. Estas estratégias podem prevenir o adoecimento crônico associado ao trabalho, assim como proteger a saúde desses trabalhadores.

Ademais, faz-se necessário o estímulo para a adoção de hábitos de vida saudáveis no cotidiano do trabalho, bem como a implementação de melhorias das condições de trabalho de modo a promover a saúde em ambientes e processos de trabalhos saudáveis.

\section{Contribuições de autoria}

Os autores contribuíram equitativamente nas diversas fases da investigação e da redação do artigo as quais incluíram o desenho do estudo, a coleta e análise dos dados, a redação e a revisão do manuscrito e a aprovação da versão final do texto para publicação e assumem integral responsabilidade pelo estudo realizado e pelo conteúdo aqui publicado.

\section{Referências}

1. Brasil. Ministério da Saúde. Departamento de Ações Programáticas Estratégicas Plano de reorganização da atenção à hipertensão arterial e ao diabetes mellitus: hipertensão arterial e diabetes mellitus. Brasília, DF: Ministério da Saúde. 2001.

2. Lima ACS, Araújo MFM, Freitas RWJF de, Zanetti ML, Almeida PC, Damasceno MMC. Fatores de risco para diabetes mellitus tipo 2 em universitários: associação com variáveis sociodemográficas. Rev Latino-Am Enferm [Internet]. 2014 [citado em 29 jan 2018];22(3):484-90. Disponível em: https://www.scielo.br/scielo.php?script =sci arttext\&pid =S0104-11692014000300484\&lng = pt\& $\mathrm{nrm}=\mathrm{iso \& t} \operatorname{lng}=\mathrm{pt}$

3. Gilbert-Ouimet M, Trudel X, Brisson C, Milot A, Vezina M. Adverse effects of psychosocial factors on blood pressure: sistematic review of studies om demand-control support and effort-reward imbalances models. Scand J Work Environ Health [Internet]. 2014 [citado em 7 out 2020];40(2),10932. Disponível em: https://www.sjweh.fi/show_ abstract.php?abstract_id=3390\&fullText $=1$

4. Eriksson AK, van den Donk M, Hilding A, Ostenson CG. Work stress, sense of coherence, and risk of Type 2 diabetes in a prospective study of middle-aged Swedish men and women. Diabetes Care [Internet]. 2013 [citado em 7 out 2020];36:2683-9, 2013. Disponível em: http://care.diabetesjournals.org/content/ diacare/36/9/2683.full.pdf

5. Brasil. Ministério da Saúde. Secretaria de Vigilância em Saúde. Departamento de Análise de Situação de Saúde. Plano de ações estratégicas para o enfrentamento das doenças crônicas não transmissíveis (DCNT) no Brasil 2011-2022 [Internet]. Brasília, DF: Ministério da Saúde; 2011 [citado em 7 out 2020]. Disponível em: http:// bvsms.saude.gov.br/bvs/publicacoes/plano_acoes enfrent_dcnt_2011.pdf

6. Steptoe A, Wardle J, Pollard TM, Canaan, L, Davies GJ. Stress, social support and health-related behavior: A study of smoking, alcohol consumption and physical exercise. J Psychosom Res [Internet]. 1996 [citado em 7 out 2020];41(2):171-180. Disponível em: https://www.sciencedirect.com/ science/article/pii/0022399996000955

7. Ng DM, Jeffery R.W. Relationships between Perceived Stress and Health Behaviors in a Sample of Working Adults. Health Psychol [Internet]. 2003 [citado em 7 out 2020];22(6):638-642. Disponível em: https://doi.apa.org/record/2003-09670014?doi $=1$ 
8. Couto HA, Vieira FLH, Lima EG. Estresse ocupacional e hipertensão arterial sistêmica. Rev Bras Hipertens [Internet]. 2007 [citado em 29 jan 2018];14(2):112-5. Disponível em: http:/departamentos.cardiol.br/dha/ revista/14-2/11-ocupacional.pdf

9. Araújo TM, Aquino E, Menezes G, Santos CO, Aguiar L. Aspectos psicossociais do trabalho e distúrbios psíquicos entre trabalhadoras de enfermagem. Rev Saúde Pública [Internet] 2003 [citado em 29 jan 2018];37(103):424-33. Disponível em: https://www.scielo.br/scielo.php?script = sci arttext\&pid $=$ S0034-89102003000400006\&lng $=\overline{\text { en }}$

10. Carvalho DB, Araújo TM, Bernardes KO. Transtornos mentais comuns em trabalhadores da Atenção Básica à Saúde. Rev Bras Saúde Ocup [Internet]. 2016 [citado em 7 out 2020];41:e17. Disponível em: http://www.scielo. br/scielo.php?script $=$ sci_arttext\&pid $=$ S030376572016000100210\&lng $=\mathrm{en}$

11. Sousa CC, Araújo TM, Lua I, Gomes MR. Occupational stress and job dissatisfaction with health work. Psicol Reflex Crít [Internet]. 2019 [citado em 7 out 2020]; 32:18. Disponível em: https://prc.springeropen.com/articles/10.1186/ s41155-019-0132-5

12. Araújo TM, Mattos AIS, Almeida MMG, Santos KOB. Aspectos psicossociais do trabalho e transtornos mentais comuns entre trabalhadores da saúde: contribuições da análise de modelos combinados. Rev Bras Epidemiol [Internet]. set 2016 [citado em 7 out 2020];19(3):645657. Disponível em: http://www.scielo.br/ scielo.php?script $=$ sci arttext\&pid $=\mathrm{S} 1415$ 790X2016000300645\&्lng=en. http://dx.doi. org/10.1590/1980-5497201600030014

13. Vilarinho RMF, Lisboa MTL. Diabetes mellitus: fatores de risco em trabalhadores de enfermagem. Acta Paul Enferm [Internet] 2010 [citado em 29 jan 2018];23(4):557-61. Disponível em: http://www. scielo.br/pdf/ape/v23n4/18.pdf

14. Karasek RAJ. Job Demands, Job Decision Latitude, and Mental Strain: Implications for Job Redesign. Adm Sci Q [Internet]. 1979 [citado em 13 nov 2017];24(2):285. Disponível em: http://www.jstor. org/stable/2392498?origin $=$ crossref
15. Araújo TM, Graça CC, Araújo E. Estresse ocupacional e saúde: contribuições do Modelo Demanda-Controle. Ciênc saúde coletiva [Internet] 2003 [citado em 29 jan 2018];8(4):991-1003. Disponível em: http://www.scielo.br/pdf/csc/v8n4/ a21v8n4.pdf

16. Greenland S, Rothman K. Introduction to Stratified Analysis In: Rothman K, Greenland S, Lasch, TL. Modern Epidemiology. 3a ed. Philadelphia: Lippincott Williams \& Wilkins; 2008. p. 258-282.

17. Alves MGM, Braga VM, Faerstein E, Lopez CS, Junger W. The demand-control model for job strain: a commentary on different ways to operationalize the exposure variable. Cad Saúde Pública [Internet]. jan 2015 [citado em 18 jun 2018];31(1):20812. Disponível em: http://www.scielo.br/scielo. php?script $=$ sci_arttext\&pid $=$ S0102-311X20150001 00208\&lng $=$ en\&nrm $=$ iso

18. Zaitune MP do A, Barros MB de A, César CLG, Carandina L, Goldbaum M. Hipertensão arterial em idosos: prevalência, fatores associados e práticas de controle no Município de Campinas, São Paulo, Brasil. Cad Saúde Pública [Internet] 2006 [citado em 29 jan 2018];22(2):285-94. Disponível em: http://www.scielo.br/pdf/csp/v22n2/06.pdf

19. Ortiz MCA, Zanetti ML. Diabetes Mellitus: fatores de risco em uma instituição de ensino na área da saúde. Rev Latino-Am Enferm [Internet]. 2000 [citado em 29 jan 2018];8(6):128-32. Disponível em: http://www.scielo.br/pdf/rlae/v8n6/12359.pdf

20. Almeida VCF, Zanetti ML, Almeida PC, Damasceno MMC. Ocupação e fatores de risco para diabetes tipo 2: estudo com trabalhadores de enfermagem. Rev Latino-Am Enferm [Internet]. 2011 [citado em 2018 Jan 29];19(3):[9 telas]. Disponível em: http://www.scielo.br/pdf/rlae/ v19n3/pt_05.pdf

21. Watson NL, VanderVeen JW, Cohen LM, DeMarree KG, Morrell HER. Examining the interrelationships between social anxiety, smoking to cope, and cigarette craving. Addict Behav [Internet]. Aug 2012 [citado em 7 out 2020];37(8):986-9. Disponível em: https://www.sciencedirect.com/science/article/abs/pii/ S0306460312001268 